\title{
RECURRENCE AND ALMOST PERIODICITY IN A GENERATIVE TRANSFORMATION GROUP
}

FRED A. ROBERSON

\begin{abstract}
A point $p$ in a transformation group $(X, T, \Pi)$ is recurrent if for every neighborhood $U$ of $p$ there is an extensive set $E \subset T$ such that $p E \subset U$. The point $p$ is almost periodic if there is a syndetic set $A \subset T$ such that $p A \subset U$. This paper proves that a recurrent point in a locally compact, generative transformation group with an equicontinuous neighborhood must also be almost periodic.
\end{abstract}

The purpose of this paper is to prove that, under certain conditions, a recurrent point must also be almost periodic. The definitions are as in the book [1]. All spaces are assumed to be Hausdorff.

THEOREM. Let $(X, T, \Pi)$ be a transformation group, where $X$ is a locally compact uniform space and $T$ is generative and equicontinuous in a neighborhood of a point $p$. If $p$ is recurrent then $p$ is almost periodic.

The proof of the theorem will make use of the following remarks, which are known for the most part.

REMARK 1. T is topologically isomorphic to $C \times I^{l} \times R^{m}$, where $I$ is the integers with the discrete topology, $R$ is the reals with the usual topology and $C$ is a compact abelian group.

REMARK 2. If $E$ is an extensive subset of $T$, then for any specified coordinates in the representation $C \times I^{l} \times R^{m}$ a sequence on $E$ can be found which increases without bound in these coordinates and decreases without bound in the others.

Proof. This follows directly from the definition of extensive.

REMARK 3. A subset $A$ of $T$ is syndetic if and only if there exists a compact subset $K$ of $T$ such that $t K \cap A \neq \varnothing$ for every $t$ in $T$.

Proof of THE THEOREM. Let $U$ be an open neighborhood of $p$ and let $A=\{t \mid t \in T, p t \in U\}$. Then $A$ is extensive and we wish to show that $A$ is syndetic. Suppose that this is not the case.

Received by the editors March 31, 1971.

AMS 1969 subject classifications. Primary 5482, 9480, 2240, 5747.

Key words and phrases. Transformation group, generative, recurrent, almost periodic, equicontinuous.

(c) American Mathematical Society 1972 
Let $U^{*}$ be an open neighborhood of $p$ such that $T$ is equicontinuous on $U^{*}$. Let $\gamma$ be an index of the uniformity $\mathscr{U}$ of $X$ such that $\operatorname{cl}(p \gamma) \subset U \cap U^{*}$ and $\operatorname{cl}(p \gamma)$ is compact. Let $\alpha$ be an index of $X$ such that $p \alpha^{2} \subset p \gamma, \operatorname{cl}(p \alpha)$ is compact, $\operatorname{cl}(p \alpha) \alpha \subset U \cap U^{*}$.

Let $W=\{t \mid t \in T, p t \in p \alpha\}$. Then $p \bar{W} \subset \operatorname{cl}(p W) \subset \operatorname{cl}(p \alpha) \subset U$ and $\bar{W}$ is extensive but not syndetic. For $\bar{W} \subset A$ and if $\bar{W}$ were syndetic, $A$ would be syndetic, which is not the case.

REMARK 4. If $K_{2 n}=C \times[0,2 n] \times \cdots \times[0,2 n]$ then there exists a $t$ in $T$ such that $t K_{2 n} \cap \bar{W}=\varnothing$.

Proof. This is the negation of Remark 3 for the compact set $K_{2 n}$.

REMARK 5. There exists a $t^{\prime}$ in $T$ such that $\operatorname{int}\left(t^{\prime} K_{2 n}\right) \cap \bar{W}=\varnothing$ and $\operatorname{Fr}\left(t^{\prime} K_{2 n}\right) \cap \bar{W} \neq \varnothing$.

Proof. This is accomplished by a translation of the set $t K_{2 n}$ obtained from Remark 4.

REMARK 6. There is a box $K_{n}^{\prime}=C \times I_{1}^{n} \times \cdots \times I_{m+l}^{n}$ where $I_{i}^{n}=[0, n]$ or $[-n, 0]$, and an $s_{n}$ in $\bar{W}$, such that int $\left(s_{n} K_{n}^{\prime}\right) \cap \bar{W}=\varnothing, \operatorname{Fr}\left(s_{n} K_{n}^{\prime}\right) \cap \bar{W} \neq \varnothing$ and $s_{n}$ appears at one corner of the box $s_{n} K_{n}$.

Proof. This follows from Remark 5 by picking $s_{n} \in \operatorname{Fr}\left(t^{\prime} K_{2 n}\right) \cap \bar{W}$ and taking a subbox of $t^{\prime} K_{2 n}$ whose sides are of length $n$.

The purpose of the last three remarks is to establish that there are boxes in $T=C \times I^{l} \times R^{m}$ of arbitrarily long sides with an element of $\bar{W}$ in one corner and no elements of $\bar{W}$ in the interior of the boxes. We will now establish the contradiction. The boxes above are constructed in such a way that we can distinguish one corner from the other. Hence we can classify each box by which corner the element of $\bar{W}$ appears. Since there are only $2^{m+l}$ corners there must be one corner in which the element of $\bar{W}$ appears for an infinite number of the boxes. We can assume without loss of generality that there are an infinite number of boxes $s_{k} K_{k}^{\prime}$ where $K_{k}^{\prime}=C \times$ $[0, k] \times \cdots \times[0, k]$ and $s_{k} \in \bar{W}$. Since $s_{k} \in \bar{W}, p s_{k}$ is in $\operatorname{cl}(p \alpha)$ and therefore has a limit point $q$. Since $\operatorname{cl}(p \alpha)$ is compact there exists a subnet $\left\{N_{m} \mid m \in D\right\}$ of $\left\{p s_{k}\right\}$ such that $\left\{N_{m} \mid m \in D\right\} \rightarrow q$. Let $\beta$ be an index of $X$ such that $\beta \circ \beta \circ \beta \subset \alpha$ and, since $q$ is an equicontinuous point, there is a neighborhood $V$ of $q$ such that $x$ in $V$ implies $(x t, q t) \in \beta$ for all $t$ in $T$. Now there is an $n$ in $D$ such that $p s_{k_{m}}$ is in $V$ for all $m \geqq n$. From Remark 2 there is an $r$ in $T$ such that $p\left(s_{k_{n}}+r\right)$ is in $p \beta$ and every coordinate of $r$ is positive. Hence

$$
\begin{aligned}
\left(p, p\left(s_{k_{m}}+r\right)\right) & =\left(p, p\left(s_{k_{n}}+r\right)\right) \circ\left(p\left(s_{k_{n}}+r\right), q r\right) \circ\left(q r, p\left(s_{k_{m}}+r\right)\right) \\
& \in \beta \circ \beta \circ \beta \subset \alpha,
\end{aligned}
$$

so that $s_{k_{m}}+r \in \bar{W}$ for all $m \geqq n$. But if we choose an $m \geqq n$ such that $k_{m}$ is strictly larger than the maximum coordinate of $r$ then $s_{k_{m}}+r$ also belongs to int $\left(s_{k_{m}} K_{k_{m}}^{\prime}\right)$, which is a contradiction. Therefore $\bar{W}$ is syndetic and $p$ is an almost periodic point. 


\section{REFERENCES}

1. W. H. Gottschalk and G. A. Hedlund, Topological dynamics, Amer. Math. Soc. Colloq. Publ., vol. 36, Amer. Math. Soc., Providence, R.I., 1955. MR 17, 650.

Department of Mathematics, Florida State University, Tallahassee, Florida 32306 